\title{
FACTORES PSICOEDUCATIVOS Y MOTIVACIONALES ASOCIADOS A LA ACTIVIDAD FÍSICA EN JÓVENES ADULTOS
}

\author{
Miguel Ángel Durán-Vinagre \\ Dpto. Psicología y Antropología. Universidad de Extremadura \\ mduranv@unex.es \\ Susana Sánchez Herrera \\ Dpto. Psicología y Antropología. Universidad de Extremadura \\ ssanchez@unex.es \\ Lucía Pérez Vera \\ Dpto. Psicología y Antropología. Universidad de Extremadura \\ luciapv@unex.es
}

Recepción Artículo: 27 octubre 2021

Admisión Evaluación: 27 octubre 2021

Informe Evaluador 1: 28 octubre 2021

Informe Evaluador 2: 29 octubre 2021

Aprobación Publicación: 30 octubre 2021

\section{RESUMEN}

La literatura científica muestra un amplio abanico sobre los factores que influyen en los adolescentes, especialmente en relación con la actividad física, pero, sin embargo, no lo es tanto en la literatura especializada con otros aspectos, como con los factores psicoeducativos. Por ello, el objetivo principal de la revisión sistemática que presentamos en este trabajo revisión de la literatura de estudios empíricos que evalúan la relación entre motivación y actividad física como factores psicoeducativos para la mejora de los hábitos saludables en los adolescentes a partir de las bases de datos Web Of Science (WOS) y SCOPUS en un periodo de diez años comprendido entre 2010-2019, considerando la metodología PRISMA. Se utilizaron diferentes palabras claves: "Physical Activity" AND "Motivation" AND "Information Technology" OR "Web 2.0 Technologies" AND "Adolescents". De los documentos obtenidos en las distintas bases de datos, podemos extraer como resultados que las evidencias científicas justifican que la motivación y el uso de las TIC son factores que inciden sobre la realización de actividad física. Entre las principales razones se destaca el interés existente en la sociedad por el cuidado de la salud y el aumento de la calidad de vida a consecuencia de los altos niveles de obesidad y sobrepeso, ya que el uso inapropiado de los recursos tecnológicos es el principal causante del sedentarismo, la inactividad física y la declinación de la calidad de vida de la población. Por tanto, esto presenta una estrecha relación con los factores psicoeducativos de estos jóvenes.

Palabras clave: motivación; actividad física; factores psicoeducativos; TIC; adolescentes; análisis bibliométrico 


\section{FACTORES PSICOEDUCATIVOS Y MOTIVACIONALES ASOCIADOS A LA ACTIVIDAD FÍSICA EN JÓVENES ADULTOS}

\section{ABSTRACT}

Psychoeducational and motivational factors associated with physical activity in young adults. The scientific literature shows a wide range of factors that influence adolescents, especially in relation to physical activity, but, nevertheless, it is not so much in the specialized literature with other aspects, as with psychoeducational factors. Therefore, the main objective of the systematic review that we present in this work is to review the literature of empirical studies that evaluate the relationship between motivation and physical activity as psychoeducational factors for the improvement of healthy habits in adolescents from the bases of Web of Science (WOS) and SCOPUS data in a ten-year period between 2010-2019, considering the PRISMA methodology. Different keywords were used: "Physical Activity" AND "Motivation" AND "Information Technology" OR "Web 2.0 Technologies" AND "Adolescents". From the documents obtained in the different databases, we can extract as results that the scientific evidence justifies that the motivation and the use of ICT are factors that affect the performance of physical activity. Among the main reasons is the interest in society for health care and the increase in quality of life because of high levels of obesity and overweight, since the inappropriate use of technological resources is the main cause sedentary lifestyle, physical inactivity and the decline in the quality of life of the population. Therefore, this is closely related to the psychoeducational factors of these young people.

Keywords: motivation; physical activity; psychoeducational factors; TIC; teenagers; bibliometric analysis.

\section{INTRODUCCIÓN}

Enmarcado dentro del área temática de salud, psicología y educación, podemos decir que este trabajo, en los últimos años, las investigaciones sobre la actividad física y la salud han aumentado considerablemente encontrando estudios que demuestran que la práctica regular y continuada de actividad física presenta una relación directa entre la mejora de la salud y el incremento de la esperanza de vida (Cruz-Santos, González- Rivera y Rosario-Rodríguez, 2019; Kohl y Cook, 2013; Lavie et al., 2019; Rippe, 2019). A pesar de las evidencias científicas que muestran dicha relación, la inactividad física se ha convertido en uno de los principales problemas de la sociedad actual tanto en el contexto nacional como en el internacional (García, 2019; Kohl et al., 2012; Pérez, 2014), encontrando la existencia de estudios que demuestran que son bajos los porcentajes de los adolescentes que realizan actividad física (Chuliá et al., 2005; Durán- Vinagre, Sánchez y Feu, 2019; Pérez, Requena y Zubiaur, 2005).

Relacionando esta primera información, la etapa de la adolescencia se considera un periodo de transición de la niñez a la edad adulta y se caracteriza por ser una etapa en la que se producen múltiples cambios biológicos, psicológicos y sociales (Güemes- Hidalgo, Ceñal y Hidalgo, 2017; Jaworska y MacQueen, 2015). También, constituye un periodo significativo en la configuración de los estilos de vidas relacionados con la creación de hábitos saludables (Alves de Sousa, Gall da Silva y Ferreira, 2014; Holmberg et al., 2018). A este respecto, la realización de actividad física durante esta etapa es un indicador clave para establecer prácticas de vida saludable, destacando la disminución de los niveles de sedentarismo e inactividad física en los adolescentes (Kumar, Robinson y Till, 2015; Menéndez y González, 2019; Varo, Martínez y Martínez, 2003).

Por otra parte, y con fin de dar explicación a los datos, la actividad física se ha relacionado con múltiples factores psicológicos, en los que destaca la motivación. Éste juega un papel imprescindible para practicar y promover la actividad física en la ciudadanía, debido a que el ser humano se comporta en función a una serie de conductas motivacionales a la hora de lograr metas y objetivos en diferentes contextos (Almagro, Navarro, Paramio y Sáenz-López, 2015).

En este contexto la motivación presenta una estrecha relación con las nuevas tecnologías, ya que es un recurso potenciador de la vida saludable y un factor condicionante de la actividad física. En este sentido, el uso de las aplicaciones en dispositivos móviles ha contribuido a un incremento en la evaluación de la salud (Fanning et al., 2012), encontrando diferentes aplicaciones que permiten combinar los objetivos de salud con un sistema de seguimiento (Schoeppe et al., 2016). 


\section{OBJETIVOS DE LA INVESTIGACIÓN}

El propósito del presente trabajo fue analizar la evolución del conocimiento científico producido de la relación entre motivación y actividad física como factores psicoeducativos para la mejora de los hábitos saludables en los adolescentes a partir de las bases de datos Web Of Science (WOS) y SCOPUS en un periodo de diez años comprendido entre 2010-2019.

\section{MATERIALES Y MÉTODOS}

Este tipo de estudios se enmarcan en los modelos de investigación de corte descriptivo (Cubo et al., 2011), considerándose, además, un diseño de investigación de tipo teórico (Ato, López y Benavente, 2013).

Se utilizó la metodología PRISMA para el desarrollo de la investigación (Preferred Reporting Items for Systematic reviews and Meta-Analyses) (Moher et al., 2010; 2015), formada por cuatro fases: (a) identificación, (b) selección, (c) elegibilidad e (d) inclusión. Por tanto, estas fases hacen alusión a las características de investigación que se derivan de la propuesta metodológica siendo: (a) definición de los objetivos, metodología explícita y reproducible; (b) búsqueda sistemática de evidencia siguiendo los criterios de elegibilidad; (c) evaluación de la validez de los hallazgos; y (d) presentación sistemática y síntesis de las características y hallazgos de los estudios incluidos.

\section{Estrategia de búsqueda}

La búsqueda del manuscrito se realizó en las bases de datos Web of Science (WOS) y SCOPUS utilizando palabras clave, operadores booleanos y opciones de búsqueda avanzada. Para dicha búsqueda se consideraron Ios siguientes términos y booleanos localizados en el Tesauro de la base de datos Educational Resource Information Center (ERIC) siendo: (Physical Activity) AND (Motivation) AND (Information Technology) OR (Web 2.0 Technologies) AND (Adolescents). Las palabras clave fueron utilizadas junto con los operadores lógicos "AND" y "OR".

\section{Criterios de inclusión y exclusión}

Los criterios de inclusión para los documentos fueron: (1) contener datos relevantes sobre motivación y el uso de las TIC relacionados con la actividad física; (2) realizado por adolescentes; (3) el período de publicación comprende entre 2010-2019; (4) documentos escritos en inglés o español; (5) el documento es un artículo; (6) acceso abierto del documento. Los estudios se excluyeron si: (1) el documento es distinto a un artículo; (2) incluían otros grupos de edad distintos a los adolescentes; (3) inaccesibilidad a la versión de texto completo; (4) no incluían datos relevantes para este estudio.

\section{RESULTADOS ALCANZADOS}

La búsqueda inicial identificó 218 resultados en las bases de datos descritas anteriormente: WOS y SCOPUS. Estos datos se exportaron al software de administrador de referencias (EndNote Online) y se procedió a eliminar aquellos registros duplicados siendo, en este caso, 2 estudios suprimidos. De los 216 documentos restantes, 80 de ellos son de acceso abierto, excluyendo 136 tras aplicar dicho filtro. Se leyó el texto completo de los 60 registros de acceso abiertos que pertenecían al tipo de publicación, artículo. Tras realizar un análisis más profundo, 17 estudios cumplieron con los criterios de elegibilidad y se incluyeron en los resultados de esta revisión.

En el siguiente gráfico (véase Figura 1), se representan aquellos países que presentan, al menos, 3 documentos en una de las dos bases de datos utilizadas para el presente análisis. Esta selección nos permite poder contrastar el número de publicaciones existentes en sendos casos. En este sentido, hay que remarcar que el número de documentos obtenidos para cada país seleccionado se asocia a la cifra total de registros obtenidos tras las primeras búsquedas realizadas tanto en WOS como en SCOPUS, siendo de 145 y de 73 respectivamente.

Aun así, se aprecia con total claridad que el país que manifiesta el mayor número de documentos registrados es Estados Unidos, con 49 documentos de un total de 218. También, Australia y Reino Unido arrojan unos resul- 


\section{FACTORES PSICOEDUCATIVOS Y MOTIVACIONALES ASOCIADOS A LA ACTIVIDAD FÍSICA EN JÓVENES ADULTOS}

tados significativos respecto al resto de países, ya que, en este caso, sitúan a las bases de datos de WOS y SCOPUS con una frecuencia acumulada evidente de 35 y 25 respectivamente. Los países restantes presentan resultados muy similares, en los que WOS destaca frente a SCOPUS, ofreciendo un recuento más favorable en cuanto al número de publicaciones recibidas. Sin embargo, si observamos en SCOPUS el país de Irlanda es el único que ostenta un número superior de documentos publicados respecto a WOS.

Figura 1: Publicaciones en WOS y en SCOPUS conforme al país.

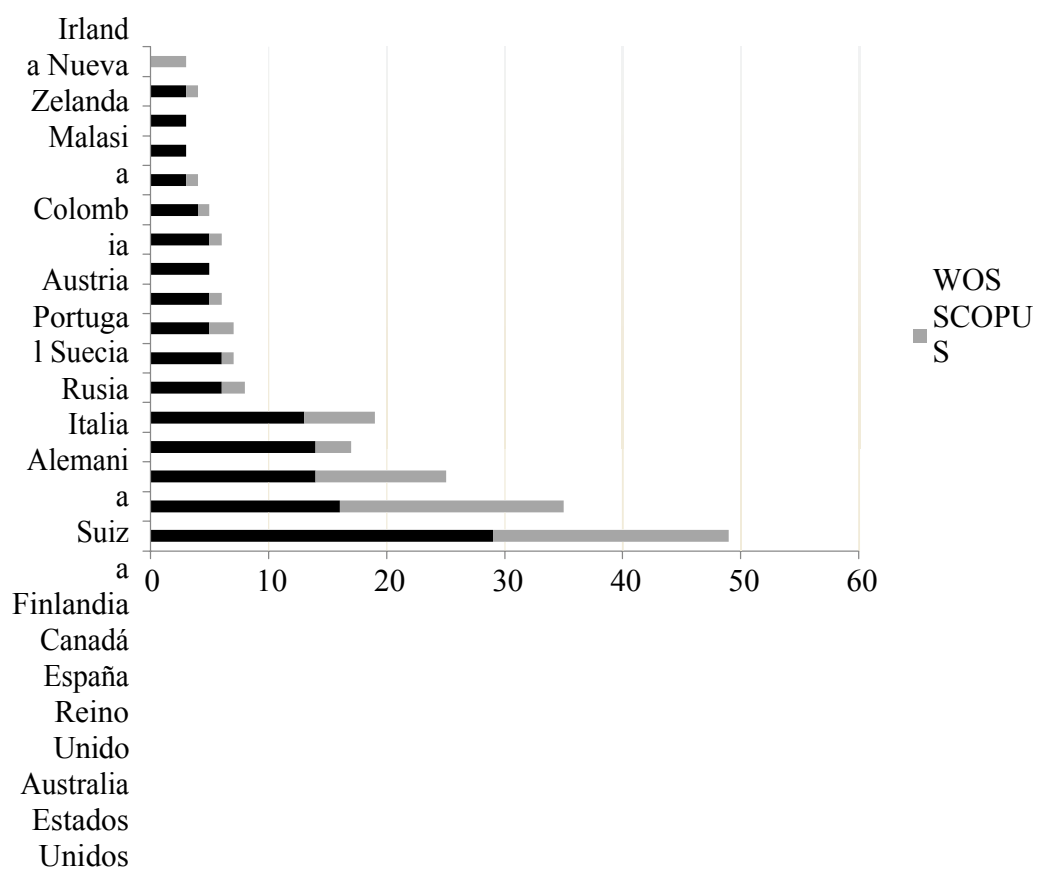

Si observamos la Tabla 1, estos resultados se ajustan a los mostrados en el gráfico anterior, destacando nuevamente Estados Unidos, ya que presenta un porcentaje mayor en WOS y en SCOPUS. Si consideramos el número de publicaciones recibidas por país según el total de documentos seleccionados, se aprecia que SCOPUS registra un porcentaje más favorable (27.4\%) que en WOS (20\%). Lo mismo ocurre si observamos Australia (SCOPUS 26\% - WOS 11\%) y Reino Unido (SCOPUS 15.1\% - WOS 9.7\%). 
Tabla 1: Descripción de porcentajes en cuanto a las publicaciones por países

\begin{tabular}{lcccc}
\hline & \multicolumn{2}{c}{ WOS } & \multicolumn{2}{c}{ SCOPUS } \\
\cline { 2 - 5 } & $\begin{array}{c}\text { Frecuenc } \\
\text { ia }\end{array}$ & $\begin{array}{c}\% \\
(\mathrm{n}=145)\end{array}$ & $\begin{array}{c}\text { Frecuenc } \\
\text { ia }\end{array}$ & $\begin{array}{c}\% \\
(\mathrm{n}=73)\end{array}$ \\
\cline { 2 - 5 } Estados & 29 & 20 & 20 & 27.4 \\
Unidos & & & & \\
Australia & 16 & 11 & 19 & 26 \\
Reino Unido & 14 & 9.7 & 11 & 15.1 \\
España & 14 & 9.7 & 3 & 4.1 \\
Canadá & 13 & 9 & 6 & 8.2 \\
Finlandia & 6 & 4.1 & 2 & 2.7 \\
Suiza & 6 & 4.1 & 1 & 1.4 \\
Alemania & 5 & 3.4 & 2 & 2.7 \\
Italia & 5 & 3.4 & 1 & 1.4 \\
Rusia & 5 & 3.4 & 0 & 0 \\
Suecia & 5 & 3.4 & 1 & 1.4 \\
Portugal & 4 & 2.8 & 1 & 1.4 \\
Austria & 3 & 2.1 & 1 & 1.4 \\
Colombia & 3 & 2.1 & 0 & 0 \\
Malasia & 3 & 2.1 & 0 & 0 \\
Nueva & 3 & 2.1 & 1 & 1.4 \\
Zelanda & & & & \\
Irlanda & 0 & 0 & 3 & 4.1 \\
\hline
\end{tabular}

\section{DISCUSIÓN}

Los resultados obtenidos manifiestan que el país que destaca del resto y presenta un mayor número de documentos registrados en ambas bases de datos es Estados Unidos. A este país le siguen Australia y Reino Unido con una frecuencia acumulada evidente de 35 y 25 respectivamente. Según los datos se desprende que las revistas indexadas en JCR son editadas prioritariamente en Estados Unidos y Reino Unido y concentran un porcentaje más favorable de publicaciones que del resto de países (Rogel-Salazar, Santiago-Batista y Martínez-Domínguez, 2017). Una de las principales causas de estos resultados se debe a que el idioma de las publicaciones es en inglés (Pastor, 2015). En este sentido, son escasas las revistas españolas que aparecen en WOS o SCOPUS y que se editan en castellano, contando mayormente con revistas que editan en inglés 0 en ambos idiomas (Bordons, Felipe y Gómez, 2002; Díaz et al, 2001).

Por otro lado, es evidente que un aspecto muy importante a la hora de evaluar la situación de la producción científica es su grado de internacionalización (Chinchilla- Rodríguez et al., 2018; González, Valderrama y Benavent, 2012; Ibarra, Castro y Barrenechea, 2007). En este sentido se han realizado colaboraciones con autores de otros países, en los que destaca Estados Unidos con un (7.1\%), seguido de Reino Unido, Italia, Francia y Alemania. Si consideramos las cifras de citas y el factor de impacto, se observa que las publicaciones en colaboración internacional tienen un impacto más alto (4.47 citas por artículo) que las que solo tienen autores españoles (3.63) (Ruiz, 2012).

\section{CONCLUSIONES}

Las TICs se han empleado como recurso para mejorar la motivación hacia la práctica de actividad física. Los nuevos recursos electrónicos se están diseñando para proporcionar un acceso interactivo entre diferentes dispo- 


\section{FACTORES PSICOEDUCATIVOS Y MOTIVACIONALES ASOCIADOS A LA ACTIVIDAD FÍSICA EN JÓVENES ADULTOS}

sitivos y programas que permiten al usuario conocer su nivel de práctica en diferentes contextos e interactuar con otras personas.

Son necesarios estudios que analicen en los adolescentes estos factores y variables, ya que es una etapa en la que se configuran y presentan cambios importantes. Aunque para esta comunicación nos hayamos centrado en los resultados por países, se deja entrever sobre el tópico analizado que es necesario realizar investigaciones que asocien el uso de recursos tecnológicos y el tipo feedback arrojado a través de los mensajes motivadores hacia la práctica y/ o las recompensas que ofrecen las apps y sus beneficios sobre la realización de A.F. Esta actividad debería ser medida de la forma más objetiva posible valorando sus efectos sobre la condición física. Las tecnologías pueden ayudar al profesorado a potenciar la práctica de actividad física fuera del contexto formal del aula, favoreciendo la autonomía de los jóvenes para controlar su actividad y participar en nuevos entornos de práctica físico-deportiva. Los docentes adquieren un rol importante en las distintas etapas educativas para enseñar y promover las ventajas de las tecnologías en el fomento y control de la actividad física como mediadores para la mejora de hábitos y estilos de vida más saludables. Así pues, a modo de síntesis, se observa que la relación entre motivación y actividad física son aspectos y elementos que se asocian con los factores psicoeducativos a la hora de mejorar los hábitos de una persona.

\section{REFERENCIAS BIBLIOGRÁFICAS}

Almagro, B. J., Navarro, I., Paramio, G., y Sáenz-López, P. (2015). Consecuencias de la motivación en las clases de Educación Física. Revista Digital de Educación Física, 6(34), 26-41.

Alves de Sousa, Z. A., Gall da Silva, J., y Ferreira, M. A. (2014). Knowledge and practices of teenagers about health: implications for the lifestyle and self-care. Escola Anna Nery, 18(3), 400-406.

Ato, M., López, J. J., y Benavente, A. (2013). Un sistema de clasifación de los diseños de investigación en psicología. Anales de Psicología, 29(3), 1038-1059.

Bordons, M., Fernández, M. T., y Gómez, I. (2002). Advantages and limitations in the use of impact factor measures for the assessment of reserach performance in a peripheral country. Scientometrics, 55, 196-206.

Chinchilla-Rodríguez, Z., Miao, L., Murray, D., Robinson-García, N., Costas, R., y Sugimoto, C.R. (2018). A global comparison of scientific mobility and collaboration according to national scientif capacities. Frontiers in Research Metrics and Analytics, 3, 17.

Chuliá, M., Ferrer, E., Lizama, N., Martin, S., y Monrabal, C. (2005). El sedentarismo en los jóvenes universitarios. Educare 21, 17, 125-129.

Cruz-Santos, A., González-Rivera, J. A, Rosario-Rodríguez, A. (2019). Attitudes towards physical exercise and sports: A psychometric study in college students. Revista Evaluar, 19(1), 58-72.

Cubo, S., Martín, B., y Ramos, J. L. (Coord.) (2011). Métodos de investigación y análisis de datos en ciencias sociales y de la salud. Editorial Pirámide.

Díaz, M., Asensio, B., Llorente, G., Moreno, E., Montori, A., Palomares, F., Palomo, J., Pulido, F., Senar, J. C., y Tellería, J. L. (2001). El futuro de las revistas científicas españolas: un esfuerzo científico, social e institucional. Revista Española de Documentación Científica, 24(3), 306-314.

Durán-Vinagre, M., Sánchez, S., y Feu, S. (2019). Influencia de la actividad físico - deportiva y la intención de ser físicamente activos en universitarios de la facultad de educación de la UEX. International Journal of Developmental and Educational Psychology, 5(5), 83-96.

García, W. F. (2019). Sedentarismo en niños y adolescentes: factor de riesgo en aumento. Revista Científica Mundo de la Investigación y el Conocimmiento, 3(1),1602-1624.

González, G., Valderrama, J. C., y Benavent, R. A. (2012). Análisis del proceso de internacionalización de la investigación española en ciencia y tecnología (1980- 2007). Revista española de Documentación Científica, 34(1), 94-118.

Güemes-Hidalgo, M., Ceñal, M. J., y Hidalgo, M. I. (2017). Pubertad y adolescencia. Revista de Formación 
Continuada de la Sociedad Española de Medicina de la Adolescencia, 5(5), 7-22.

Holmberg, C., Larsson, C., Korp, P., Lindgren, E. C., Jonsson, L., Fröberg, A., Chaplin, J., y Berg, C. (2018). Empowering aspects for healthy food and physical activity habits: adolescents experiences of a school-based intervention in a disadvantaged urban community. Internacional Journal of Qualitative Studies on Health and Well-being, 13(1).

Ibarra, J. A., Castro, J., y Barrenechea, J. I. (2007). La evaluación de la actividad científica en ciencias sociales y humanidades. Servicio Editorial de la Universidad del País Vasco.

Jaworska, N., y MacQueen, G. (2015). Adolescence as a unique developmental period. Journal Psychiatry Neuroscience, 40(5), 291-293.

Kohl, H. W., Craig, C. L., Lambert, E. V., Inoue, S., Alkandari, J. R., Leetongin, G., y Kahlmeier, S. (2012). The pandemic of physical inactivity: global action for public health. Lancet, 380(9838), 294-305.

Kohl, H. W., y Cook, H. D. (2013). Educating the student body. Taking physical activity and physical education to school. The National Academies Press. Washington.

Kumar, B., Robinson, R., y Simon, T. (2015). Physical activity and heath in adolescence. Clinical Medicine, 15(3), 267-272.

Lavie, C. J. Ozemek, C., Carbone, S., Katzmarzyk, P. T., y Blair, S. N. (2019). Sedentary behavior, exercise, and cardiovascular health. Circulation Research, 124(5).

Menéndez, D., y González, C. (2019). Relaciones entre la práctica de actividad física y deportiva, el autoconcepto, la imagen corporal y los hábitos alimentarios en estudiantes de primaria. E-balonmanocom: Revista de Ciencias del Deporte, 15(1), 79-96.

Moher, D., Liberati, A., Tetzla, J., y Altman, D. G. (2010). Preferred reporting items for systematic reviews and meta-analyses: The PRISMA statement. Int. J. Surg, 8, 336-341.

Moher, D., Shamseer, L., Clarke, M., Ghersi, D., Liberati, A., Petticrew,M., ... Group,

P. P. (2015). Preferred reporting items for systematic review and meta-analysis protocols (PRISMA-P) 2015 statement. Systematic Reviews, 4(1), 1-9.

Pastor, J. M. (2015). Revistas del Journal Citation Reports sobre Comunicación. ZER - Revista de Estudios de Comunicación, 22(42), 211-233.

Pérez, B. M. (2014). Salud: entre la actividad física y el sedentarismo. Anales Venezolanos de Nutrición, 27(1), 119-128.

Pérez, D., Requena, C., y Zubiaur, M. (2005). Evolución de motivaciones, actitudes y hábitos de los estudiantes de la facultad de ciencias de la actividad física y del deporte de la Universidad de León. Motricidad European Journal of Human Movement, 14, 65-79.

Rippe, J. M. (2019). Lifestyle Medicine 2019: deeper, broader, and more precise. American. Journal of Lifestyle medicine, 13(5), 436-439.

Rogel-Salazar, R., Santiago-Bautista, I., y Martínez-Domínguez, N. (2017). Revistas científicas latinoamericanas de Comunicación indizadas en WOS, Scopus y bases de datos de Acceso Abierto. Comunicación y Sociedad, (30), 167-196.

Ruiz, F. (2012). La investigación en informática en espana. análisis bibliométrico.

Novatica, 215, 54-58.

Schoeppe, S., Alley, S., Van Lippevelde, W., Bray, N. A., Williams, S. L., Duncan, M. J., y Vandelanotte, C. (2016). Efficacy of interventions that use apps to improve diet, physical activity and sedentary behaviour: a systematic review. International Journal of Behavioral Nutrition and Physical Activity, 13(1), 127. 
